\title{
The equation of state of neutron matter, symmetry energy, and neutron star structure
}

\author{
S. Gandolfi ${ }^{1}$, J. Carlson ${ }^{1}$, S. Reddy ${ }^{2}$, A. W. Steiner ${ }^{2}$, and R. B. Wiringa ${ }^{3}$ \\ 1 Theoretical Division, Los Alamos National Laboratory Los Alamos, NM 87545 \\ 2 Institute for Nuclear Theory, University of Washington, Seattle, WA 98195 \\ 3 Physics Division, Argonne National Laboratory, Argonne, IL 60439
}

the date of receipt and acceptance should be inserted later

\begin{abstract}
We review the calculation of the equation of state of pure neutron matter using quantum Monte Carlo (QMC) methods. QMC algorithms permit the study of many-body nuclear systems using realistic two- and three-body forces in a nonperturbative framework. We present the results for the equation of state of neutron matter, and focus on the role of three-neutron forces at supranuclear density. We discuss the correlation between the symmetry energy, the neutron star radius and the symmetry energy. We also combine QMC and theoretical models of the three-nucleon interactions, and recent neutron star observations to constrain the value of the symmetry energy and its density dependence.
\end{abstract}

PACS. XX.XX.XX No PACS code given

\section{Introduction}

Knowledge of the equation of state (EoS) of pure neutron matter at and above nuclear saturation density is the important bridge between the symmetry energy in nuclei and neutron star properties. Its accurate calculation presents a challenge because of the strong interactions between neutrons and due to the large extrapolation in isospin necessary to use experimental constraints from nuclear structure. In recent years a quantum Monte Carlo (QMC) technique, called auxiliary field diffusion Monte Carlo (AFDMC), has been developed to study large pure neutron systems with the same accuracy as Green's function Monte Carlo (GFMC), another QMC technique that has been hugely successful in calculating the ground state properties of nuclei up to 12 nucleons using realistic nuclear Hamiltonians with local two- and three-body forces. Given a Hamiltonian, the many-body system is generally solved with controlled systematic errors within $1-2 \%$. The combined use of GFMC and AFDMC is an important step forward to study the neutron matter equation of state because the calculation of light nuclei and infinite matter is now possible following the same scheme and within the same systematic uncertainties.

Because of its important role in astrophysics, the symmetry energy has received considerable attention in recent years. The symmetry energy $E_{\text {sym }}$ is given by the difference of the energy per particle in symmetric nuclear matter and pure neutron matter, and represents the energy cost of sustaining an isospin-asymmetry in the homogeneous nucleonic matter. In large nuclei and dense systems the competition between the Coulomb energy cost of hav- ing a large number of protons (and electrons) and the energy cost associated with large isospin asymmetry is key to understanding the mechanism of stability and neutronskin thickness of very neutron-rich nuclei in the laboratory, the reaction pathways and abundances of heavy neutron-rich nuclei produced during r-process nucleosynthesis, neutron star structure and many related phenomena occurring in neutron stars and supernova 1 .

In this paper we will review a recent AFDMC calculation of the equation of state of neutron matter using realistic models 1 of two and three nucleon interactions and discuss constraints to the symmetry energy obtained from neutron stars observations.

\section{Nuclear Hamiltonian}

In our model, neutrons are non-relativistic point particles interacting via two- and three-body forces:

$$
H=\sum_{i=1}^{A} \frac{p_{i}^{2}}{2 m}+\sum_{i<j} v_{i j}+\sum_{i<j<k} V_{i j k} .
$$

where $m$ is the neutron mass, and $v_{i j}$ and $V_{i j k}$ are two and three-body potentials. Because nucleons are composite particles, many-body forces are natural and their relative importance increases with density. The two-body potential that we use is the Argonne $\mathrm{AV}^{\prime}$, a simplified

\footnotetext{
1 We define a realistic potential as one that can reproduce measured nucleon-nucleon scattering phase shifts and properties of light nuclei.
} 
form of the Argonne AV18 2. This potential has been obtained by fitting nucleon-nucleon scattering data up to laboratory energies of $350 \mathrm{MeV}$ with very high precision. It has to be noted, however, that it also reproduces many elastic phase shifts up to much higher energies, as shown in Fig. 1. At saturation density, two neutrons on the Fermi surface with momenta $\pm k_{F}\left(\left|k_{F}\right| \simeq 330 \mathrm{MeV}\right)$ have a center of mass energy of $\sim 120 \mathrm{MeV}$, which would correspond to a laboratory energy of $\sim 240 \mathrm{MeV}$.

It has also been shown that this two-nucleon potential, when combined with a model of the three-nucleon interaction, reproduces low energy properties of light nuclei with high precision 3. Four- and higher-body forces are generally found to be negligible. In more systematic approaches, based on chiral perturbation theory, a hierarchy of many-body forces arises naturally [4 and explicit recent calculations of neutron matter in the vicinity of nuclear saturation density have shows that the contribution from four-body forces is very small [5,6].

The two-body Argonne potential is written as a sum of operators:

$$
v_{i j}=\sum_{p=1}^{M} v_{p}\left(r_{i j}\right) O^{(p)}(i, j),
$$

where $O^{(p)}(i, j)$ are spin-isospin dependent operators. The number of operators $M$ characterizes the interaction; the most accurate of them is the Argonne AV18 with $\mathrm{M}=18$ [2]. Here we consider a simpler form AV8' , which is a reprojection of the S- and P-wave parts of AV18 onto eight operators; the difference between this simpler form and the full AV18 potential can be computed perturbatively 7 . It has been shown that the difference between AV18 and $\mathrm{AV}^{\prime}$ is less than $0.25 \%$ in pure neutron systems [8].

The eight $O^{(p)}(i, j)$ terms in $\mathrm{AV}^{\prime}$ are given by:

$$
O^{p=1,8}(i, j)=\left(1, \boldsymbol{\sigma}_{i} \cdot \boldsymbol{\sigma}_{j}, S_{i j}, \mathbf{L}_{i j} \cdot \mathbf{S}_{i j}\right) \times\left(1, \boldsymbol{\tau}_{i} \cdot \boldsymbol{\tau}_{j}\right),
$$

where the operator $S_{i j}=3 \boldsymbol{\sigma}_{i} \cdot \hat{r}_{i j} \boldsymbol{\sigma}_{j} \cdot \hat{r}_{i j}-\boldsymbol{\sigma}_{i} \cdot \boldsymbol{\sigma}_{j}$ is the tensor operator and $\mathbf{L}_{i j}=-\imath \hbar \boldsymbol{r}_{i j} \times\left(\boldsymbol{\nabla}_{i}-\boldsymbol{\nabla}_{j}\right) / 2$ and $\mathbf{S}_{i j}=\hbar\left(\boldsymbol{\sigma}_{i}+\boldsymbol{\sigma}_{j}\right) / 2$ are the relative angular momentum and the total spin for the pair $i j$. For neutrons $\boldsymbol{\tau}_{i} \cdot \boldsymbol{\tau}_{j}=1$, and we are left with an isoscalar potential. The above operators are sufficient to exactly reproduce the chargeindependent average of the AV18 phase shifts in all S- and $\mathrm{P}$-waves and all deuteron properties.

The Argonne two-body force is fitted to scattering data and correctly gives the deuteron binding energy, but for nuclei with 3 or more nucleons it is not sufficient to describe the ground state of light nuclei. It is commonly accepted that a three-body interaction is essential to overcome the underbinding of nuclei with more than two nucleons. The Urbana-IX (UIX) potential has been introduced to correct this limitation of the Argonne force. It includes the Fujita-Miyazawa term that describes the p-wave exchange of two pions between three nucleons, where the intermediate state has one nucleon excited to a $\Delta$. This term is the longest-range three-nucleon interaction and a very similar term also arises as the leading three-nucleon contribution (at $\mathrm{N}^{2} \mathrm{LO}$ ) in chiral perturbation theory [4. In addition, we add a simple short-distance phenomenological three-nucleon interaction which is spin-isospin independent to soak up short distance physics 9. The UIX force was originally proposed in combination with the Argonne AV18 and AV8' [10]. Although this model of the three-nucleon force does not fully alleviate the underbinding problem in light nuclei, it has been extensively used to study the equation of state of nuclear and neutron matter $11,12,13,14$.

Other phenomenological models of three-body force, such as the Illinois forces, have been obtained by fitting the binding energies of light nuclei up to $A \leq 8$ [15]. The most recent is the Illinois-7 (IL7) [16 which was fit to 20 states up to $\mathrm{A}=10$. It reproduces nuclear energies of 50 states (not including isobaric analogs) up to $A=12$ with an $\mathrm{rms}$ error of $600 \mathrm{keV}$. In addition to the Fujita-Miyazawa term the Illinois models also include s-wave two-pion-exchange and also three-pion-exchange ring diagrams. Such a general form for the Hamiltonian has been shown to describe the spectrum of light nuclei [15,17, n- $\alpha$ scattering [18, and high-momentum components of nuclear wave functions as observed in $\left(e, e^{\prime} p N\right)$ reactions [19].

However, the three-pion rings included in the Illinois forces leads to a very strong attraction in pure neutronmatter [20,21] and makes the EoS too soft to be compatible with observed neutron star masses and radii. This is a sign of important deficiencies in our model of the threenucleon forces, which will become especially important at higher density and larger isospin asymmetry. While chiral effective field theory ( $\chi \mathrm{EFT}$ ) provides a unified and systematic description of many-nucleon forces consistently with two-nucleon force, its regime of validity is restricted to relatively low density in the neutron star context. To access supranuclear densities, we currently need to rely on model Hamiltonians, and the specific one we discuss here must be viewed as a minimal model whose validity can in principle be tested by a combination of terrestrial experiments and astrophysical observations.

Extensions of the model abound, and a large class of three-nucleon potentials with different spin, isospin and radial dependencies must be studied to draw definite conclusions. In the future, it is hoped that new developments in effective field theory of nucleon-nucleon interactions [22], and lattice QCD will both provide useful insights on the structure of three- and four-nucleon interactions. Here, we attempt to the extract the error associated with poorly parametrized short-distance physics by varying the threenucleon force. Results obtained with relatively large variations of the coupling strengths and effective ranges nonetheless show only modest sensitivity to the details.

\section{The AFDMC method}

QMC methods provide a very useful tool to study the many-body ground state of strongly correlated systems without the need of using perturbative techniques. The advantage of QMC methods is that the calculation can 

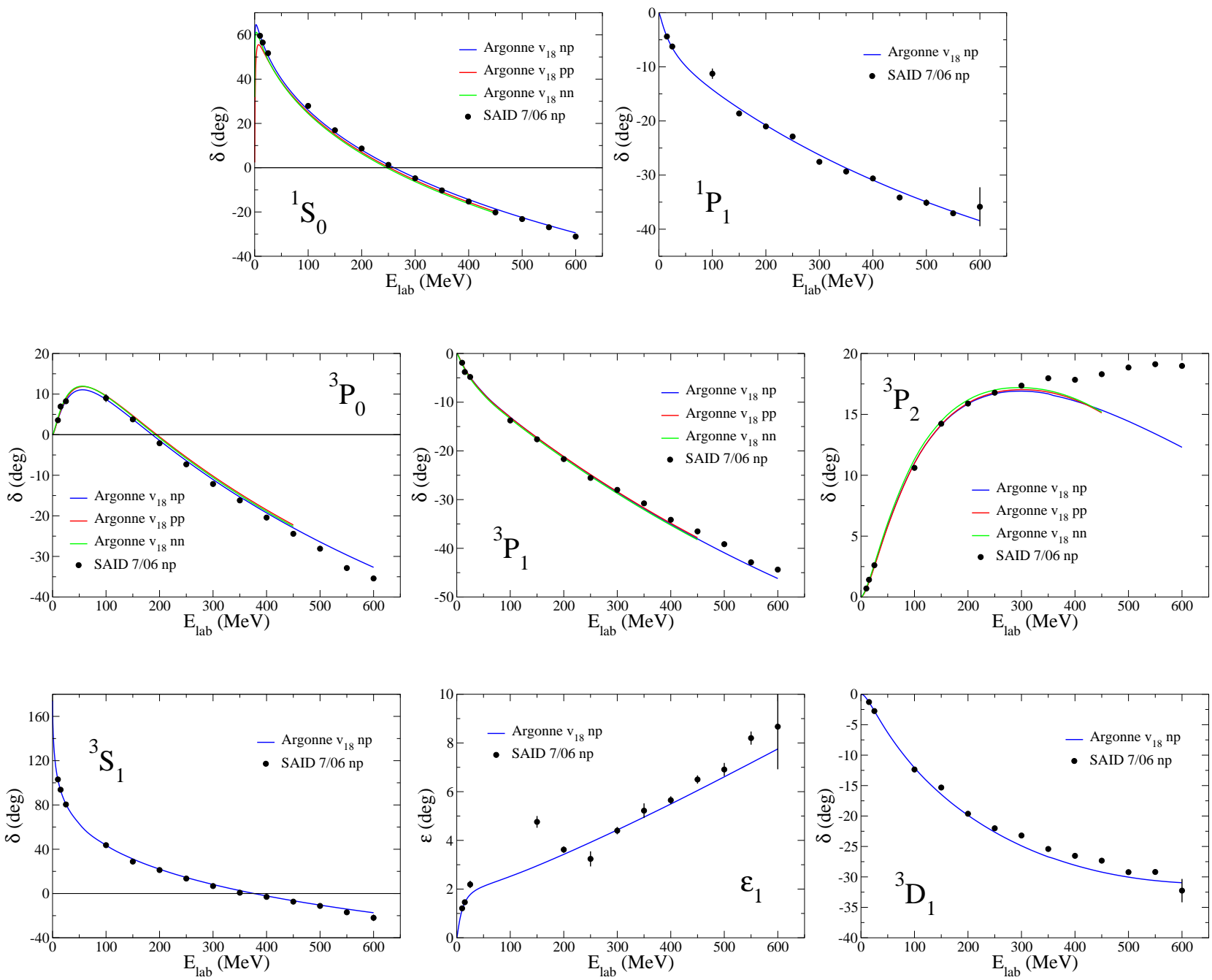

Fig. 1. Phase shifts of AV18 nucleon-nucleon potential. Experimental phase shifts are from the SAID Partial-Wave Analysis Facility (gwdac.phys.gwu.edu).

be done using a nuclear Hamiltonian with strong nonperturbative interactions that can describe high momentum observables (scattering phase shifts at large center-of-mass energy, nuclear response at large momentum transfer, and EoS of dense matter).

We solve the many-body ground-state using the AFDMC method originally introduced by Schmidt and Fantoni 23. The main idea of QMC methods is to evolve a many-body wave function in imaginary time:

$$
\Psi(\tau)=\exp [-H \tau] \Psi_{v},
$$

where $\Psi_{v}$ is a variational ansatz that includes short-range two-body correlations, and $H$ is the Hamiltonian of the system. In the limit of $\tau \rightarrow \infty, \Psi$ approaches the groundstate of $H$. The evolution in imaginary time is performed by sampling configurations of the system using Monte Carlo techniques, and expectation values are evaluated over the sampled configurations.
Technically, the wave function is represented as a set of configurations called "walkers":

$$
\langle\boldsymbol{R} \mid \Psi\rangle=\Psi(\boldsymbol{R})=\sum_{i}\left\langle\boldsymbol{R} \mid \boldsymbol{R}_{i}\right\rangle\left\langle\boldsymbol{R}_{i} \mid \Psi\right\rangle
$$

where $\boldsymbol{R}=\left\{\boldsymbol{r}_{1} \ldots \boldsymbol{r}_{n}\right\}$ are the coordinates of the system, and the propagation in imaginary time $\tau$ is obtained by solving

$$
\Psi(\boldsymbol{R}, \tau)=\int G\left(\boldsymbol{R}, \boldsymbol{R}^{\prime}, \tau\right) \Psi\left(\boldsymbol{R}^{\prime}, 0\right) d \boldsymbol{R}^{\prime}
$$

The many-body Green's Function $G\left(\boldsymbol{R}, \boldsymbol{R}^{\prime}, \delta \tau\right)$ is accurately known in the limit of small $\delta \tau$, and then the above integration can be solved using many small time steps. For spin-isospin independent interactions it is easy to write

$$
e^{-H \delta \tau} \sim e^{-V(\boldsymbol{R}) \delta \tau} G_{0}\left(\boldsymbol{R}, \boldsymbol{R}^{\prime}, \delta \tau\right),
$$


where $G_{0}$ is the free particle propagator that can be easily sampled as a particle diffusion step. The central potential is diagonal in the spin and coordinates of particles, and can be interpreted as a probability factor.

For spin-isospin dependent or non-local potentials, the factor $\exp (-V(\boldsymbol{R}) \delta \tau)$ cannot be interpreted as a simple weight factor, but needs extra care. In the GFMC all the spin-isospin states of the nucleons are explicitly included in the wave function, and the potential has the effect of changing the amplitudes of each state [24]. The idea of AFDMC is instead to rewrite the spin-dependent part of the potential as

$$
V=\sum_{i<j} \sigma_{i \alpha} A_{i \alpha, j \beta} \sigma_{j \beta}=\frac{1}{2} \sum_{n=1}^{3 N} \lambda_{n} O_{n}^{2},
$$

where $\lambda_{n}$ are eigenvectors of the matrix $A_{i \alpha, j \beta}$, and the $O_{n}$ operators are linear combinations of eigenvectors $\psi_{n}$ and spin operators and acting over particles:

$$
O_{n}=\sum_{i} \sigma_{i \alpha} \psi_{n}^{i \alpha}
$$

Using Eq. 8) it is possible to linearize the spin operators in the propagator $\exp (-V(\boldsymbol{R}) \delta \tau)$ using the HubbardStratonivich transformation

$$
e^{-\frac{1}{2} \lambda_{n} O_{n}^{2} \delta \tau}=\frac{1}{\sqrt{2 \pi}} \int_{-\infty}^{+\infty} d x_{n} e^{-\frac{x_{n}^{2}}{2}-\sqrt{-\lambda_{n} \delta \tau} x_{n} O_{n}} .
$$

The new variables $x_{n}$ are called auxiliary fields, and can be sampled using a Gaussian distribution. The effect of the $O_{n}$ (linear) operators is to rotate the single-particle spinors, and a more efficient wave function can be used.

Note that a three-neutron force can be rewritten in a quadratic spin-operators form, and then its inclusion in the AFDMC propagator is straightforward without any approximation. For more details see for example Ref. [12] and references therein.

The Green's Function Monte Carlo (GFMC) is very accurate in the study of properties of light nuclei. The variational wave function includes all the possible spin/isospin states of nucleons and it provides a good variational ansatz to start the projection in the imaginary time. The exponential growing of this states limits the present calculations to $A \leq 12$ nuclei [17] or up to 16 neutrons 8]. Using AFDMC the calculation can be extended up to $\sim 100$ neutrons, making the simulation of homogeneous matter possible. Although the implementation of GFMC and AFDMC are quite different, they agree very well in computing the energy of neutrons in an external potential [8], and for 14 neutrons in a periodic box [12].

\section{The equation of state of pure neutron matter}

We performed simulation of 66 particles in a box imposing periodic boundary conditions. This number of fermions

\begin{tabular}{c|cc}
\hline$\rho\left[\mathrm{fm}^{-3}\right]$ & AV8 & AV8 ${ }^{\prime}+$ UIX \\
\hline 0.04 & $6.55(1)$ & $6.79(1)$ \\
0.05 & $7.36(1)$ & $7.73(1)$ \\
0.06 & $8.11(1)$ & $8.65(1)$ \\
0.07 & $8.80(1)$ & $9.57(1)$ \\
0.08 & $9.47(1)$ & $10.49(1)$ \\
0.09 & $10.12(1)$ & $11.40(1)$ \\
0.10 & $10.75(1)$ & $12.39(1)$ \\
0.11 & $11.37(1)$ & $13.39(1)$ \\
0.12 & $12.00(1)$ & $14.42(1)$ \\
0.13 & $12.64(1)$ & $15.52(1)$ \\
0.14 & $13.21(1)$ & $16.66(1)$ \\
0.15 & $13.84(2)$ & $17.87(2)$ \\
0.16 & $14.47(2)$ & $19.10(2)$ \\
0.20 & $17.11(2)$ & $24.83(3)$ \\
0.24 & $19.98(3)$ & $31.85(3)$ \\
0.28 & $23.00(3)$ & $40.09(4)$ \\
0.32 & $26.45(3)$ & $49.86(5)$ \\
0.40 & $34.06(5)$ & $74.19(5)$ \\
0.48 & $42.99(8)$ & $105.9(1)$ \\
\hline
\end{tabular}

Table 1. Equation of state of neutron matter as a function of the density for various Hamiltonians; statistical errors are shown in parentheses.

is large enough to guarantee the thermodynamic limit. Finite size effects might have two contributions, from the kinetic and the potential energy, whose effects have been carefully analyzed in previous work 20,12 . The potential energy is calculated by summing over periodic boxes as discussed in Ref. 20. In any case the nuclear potential is very short-range, and becomes almost zero well within the simulation box. The kinetic energy size effects are kept under control by simulating the system with 66 fermions. The energy of 66 non-interacting fermions is very similar to the energy of the infinite system. In Ref. [12] a careful analysis has been made using the twist average boundary conditions, that guarantee a better extrapolation to the thermodynamic limit, showing that the system is large enough. In addition, in Refs. [25,26] it has been shown that for the unitary Fermi gas, a simulation with more than 40 particles essentially describes the infinite system.

The EoS of neutron matter obtained using the AV8' twobody force alone, and combined with the UIX three-body force, is presented in Table 1 . The repulsion given by the addition of UIX is evident, and becomes negligible at low densities. The results are also shown in Fig. 2, and they are very similar to those obtained by Akmal, Pandharipande and Ravenhall using the AV18 potential [11.

When the UIX three-body force is included in the calculation, we find that the contribution of the FujitaMiyazawa term is very small in pure neutron matter, while the short-range term is the dominant one. Similar contributions of the two parts of UIX have also been calculated in Ref. 11. If instead, we calculate the expectation value of UIX using a Fermi gas wave function without correlations generated by the two- and three-body forces, we find a larger contribution of the two-pion exchange term, similar to the Hartree-Fock calculation of Ref. 27]. 


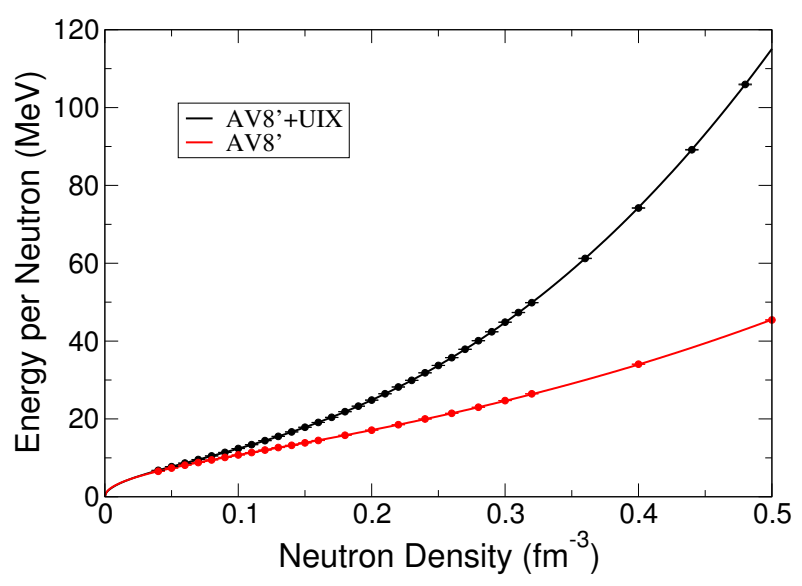

Fig. 2. The equation of state of neutron matter obtained using the $\mathrm{AV}^{\prime}$ (two-body force only) and the AV8'+UIX Hamiltonians.

We find that the EoS can be accurately parametrized using the following functional form:

$$
E\left(\rho_{n}\right)=a\left(\frac{\rho_{n}}{\rho_{0}}\right)^{\alpha}+b\left(\frac{\rho_{n}}{\rho_{0}}\right)^{\beta},
$$

where $E\left(\rho_{n}\right)$ is the energy per neutron as a function of the neutron density $\rho_{n}$, and the parameters $a, \alpha, b$, and $\beta$ are obtained by fitting the QMC results. The parametrization of the equations of state obtained with the $\mathrm{AV}^{\prime}$ and AV8'+UIX Hamiltonians are reported in Table 2

In order to address the role and the uncertainties due to the three-body force, very different models have been considered in Ref. 13. The main contribution of the threebody force is given by the short-range part, whose structure in the UIX model is

$$
V_{i j k}^{R}=A_{R} \sum_{\text {cyc }} T^{2}\left(m_{\pi} r_{i j}\right) T^{2}\left(m_{\pi} r_{j k}\right),
$$

where $m_{\pi}$ is the pion mass, and

$$
T(x)=\left(1+\frac{3}{x}+\frac{3}{x^{2}}\right) \frac{e^{-x}}{x} \xi^{2}(x) .
$$

The function $\xi(x)=1-e^{-c x^{2}}$ is a cutoff function to regularize $T(x)$ at small distances. In order to address the role of the above short-distance behavior, we have also considered very different forms to replace $T(x)$ in Eq. 12):

$$
\begin{aligned}
& f_{1}(x)=\exp (-2 \mu x), \\
& f_{2}(x)=\frac{\exp (-2 \mu x)}{\mu x}\left[1-\exp \left(-(2 \mu x)^{2}\right)\right],
\end{aligned}
$$

where the $\mu$ parameter to control the effective range of the short-range repulsion has been varied from 150 to 600 $\mathrm{MeV}$. In addition we have also replaced the general form of Eq. 12 with

$$
V_{i j k}^{R}=A_{R} f\left(r_{i j}\right) f\left(r_{j k}\right) f\left(r_{k i}\right),
$$

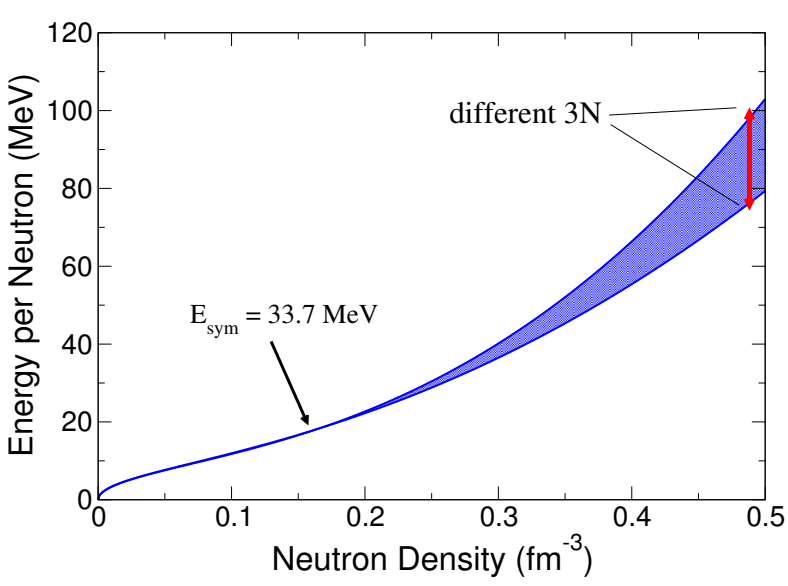

Fig. 3. The equation of state of neutron matter obtained by using various models of three-neutron force as described in the text. For each model we impose that the energy at saturation is $17.7(1) \mathrm{MeV}$.

to explore the sensitivity to the geometry of the threeneutron configuration.

For each different function $f(r)$ and different $\mu$, we have fixed the strength $A_{R}$ to reproduce a particular energy of neutron matter at saturation density $\rho_{0}=0.16$ $\mathrm{fm}^{-3}$. In this way we can relate the EoS to the symmetry energy that we shall describe in the next section.

In addition to the effects due to the short-range part, we have also explored the effect of intermediate- and longrange contributions of the three-body force. These terms, where two or three pions are exchanged between neutrons, with the creation of $\Delta$ excited states, are described in Ref. 15. We have considered different combinations of these terms with the above short-range parts in order to explore various models of three-body force with very different ranges. Also different values of the cutoff $c$ entering in Eq. 13 , and in the two- and three-pion exchange have been considered.

All the various EoS obtained with the different threeneutron forces are grouped in the band showed in Fig. 3 , and they give the same energy at saturation corresponding to $17.7(1) \mathrm{MeV}$. The various nuclear Hamiltonians give EoS with a very similar behavior up to about 0.24 $\mathrm{fm}^{-3}$, and for higher densities the result becomes modeldependent. Within our model this analysis permits us to understand the uncertainties associated with the model of the three-neutron force, but it will be important to explore the effect of other diagrams predicted by $\chi \mathrm{EFT}$ not included in the Illinois models.

As an additional investigation, all the above models of three-neutron force have also been adjusted to reproduce an energy of $16 \mathrm{MeV}$ of pure neutron matter. The results are shown in Fig. 4, where we compare with the results obtained with the AV8' and AV8' +UIX Hamiltonians. The EoS given by $\mathrm{AV}^{\prime}$ and $\mathrm{AV} 8^{\prime}+\mathrm{UIX}$ are shown without error bars because they correspond to the original Hamiltonians that we have considered. They may be considered as extreme cases because they correspond to an interval of 


\begin{tabular}{lcccccccc}
\hline $3 N$ force & $\begin{array}{c}E_{\text {sym }} \\
(\mathrm{MeV})\end{array}$ & $\begin{array}{c}L \\
(\mathrm{MeV})\end{array}$ & $\begin{array}{c}a \\
(\mathrm{MeV})\end{array}$ & $\alpha$ & $\begin{array}{c}b \\
(\mathrm{MeV})\end{array}$ & $\beta$ & $\begin{array}{c}\tilde{a} \\
(\mathrm{MeV})\end{array}$ & $\begin{array}{c}\tilde{b} \\
(\mathrm{MeV})\end{array}$ \\
\hline none & 30.5 & 31.3 & 12.7 & 0.49 & 1.78 & 2.26 & -27.4 & 6.2 \\
$V_{2 \pi}^{P W}+V_{\mu=150}^{R}$ & 32.1 & 40.8 & 12.7 & 0.48 & 3.45 & 2.12 & -28.0 & 8.8 \\
$V_{2 \pi}^{P W}+V_{\mu=300}^{R}$ & 32.0 & 40.6 & 12.8 & 0.488 & 3.19 & 2.20 & -28.2 & 8.9 \\
$V_{3 \pi}+V_{R}$ & 32.0 & 44.0 & 13.0 & 0.49 & 3.21 & 2.47 & -29.2 & 9.9 \\
$V_{2 \pi}^{P W}+V_{\mu=150}^{R}$ & 33.7 & 51.5 & 12.6 & 0.475 & 5.16 & 2.12 & -28.3 & 10.7 \\
$V_{3 \pi}+V_{R}$ & 33.8 & 56.2 & 13.0 & 0.50 & 4.71 & 2.49 & -29.7 & 12.2 \\
UIX & 35.1 & 63.6 & 13.4 & 0.514 & 5.62 & 2.436 & -29.8 & 13.6 \\
\hline
\end{tabular}

Table 2. Fitting parameters for the neutron matter EoS defined in Eqs. 111) and 21) for selected different Hamiltonians.

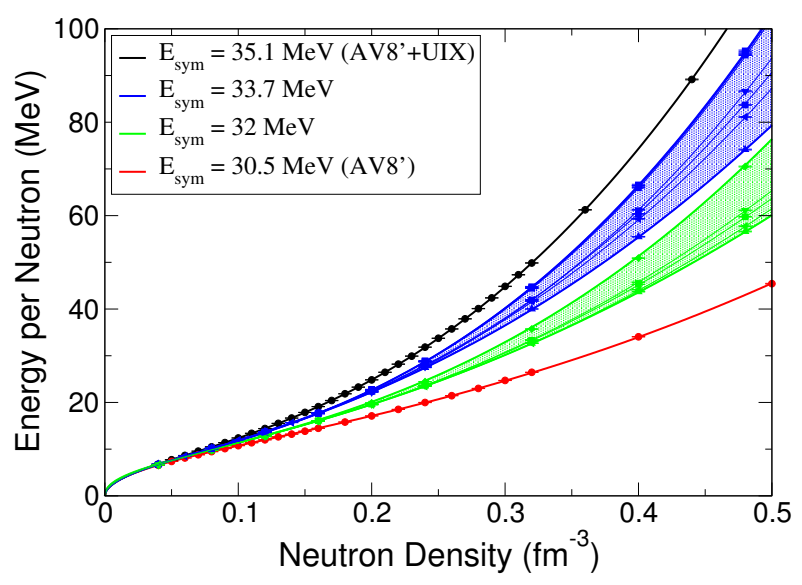

Fig. 4. The equation of state of neutron matter obtained by using various models of three-neutron force as described in the text. For each model we impose that the energy at saturation is 17.7(1) MeV (blue band), or 16.0(1) MeV (green band). The results are compared with the equations of state obtained with the $\mathrm{AV}^{\prime}$ and $\mathrm{AV} 8^{\prime}+$ UIX Hamiltonians. In the legend we indicate the corresponding symmetry energy at saturation.

the symmetry energy between 30.5 and $35.1 \mathrm{MeV}$, a range compatible with several experimental measurements [28, 29. (Error bands could be constructed by introducing a range of three-nucleon forces with these symmetry energies, but we expect the size of these error bands to be similar to the others.) To obtain a lower symmetry energy with the AV8' model of two-body force would require an attractive contribution of the three-body force. The threepion rings give attraction in pure neutron matter [21, but they would require a strong short-range repulsion to make the EoS stiff enough to support astrophysical observations.

\section{Symmetry energy}

At saturation density, the EoS of pure neutron matter is useful to extract information on the symmetry energy and its slope. The symmetry energy, $E_{\text {sym }}(\rho)$ is may be defined as the difference between the energy per baryon of pure neutron matter and the energy per baryon of infinite homogeneous nuclear matter with equal neutron number density, $\rho_{n}$, and proton number density, $\rho_{p}$. In terms of the isospin asymmetry, $x \equiv \rho_{p} / \rho$, the energy per baryon of isospin asymmetric nuclear matter can be expanded in even powers of $x$,

$E(\rho, x)=E_{0}(\rho)+E_{\mathrm{sym}}^{(2)}(\rho)(1-2 x)^{2}+E_{\mathrm{sym}}^{(4)}(1-2 x)^{4}+\ldots$,

where $E$ is the energy per baryon of the system, $E_{0}(\rho)=$ $E(\rho, x=0.5)$ is the EoS of symmetric nuclear matter, and $E_{\text {sym }}^{(4)}(\rho)$ and higher order corrections will be ignored. The symmetry energy $E_{\text {sym }}$ is thus given by

$$
E_{\text {sym }}(\rho)=E(\rho, 0)-E_{0}(\rho),
$$

where $E(\rho, 0)$ is the EoS of pure neutron matter. Near the nuclear saturation density, $\rho_{0}$, there are a number of constraints on the EoS of infinite nuclear matter from nuclear masses, charge radii, and giant resonances. The extrapolation of the binding energy of heavy nuclei to the thermodynamic limit yields $E_{0}\left(\rho_{0}\right)=-16.0 \pm 0.1 \mathrm{MeV}$ [30]. Because the pressure is zero at saturation, we can expand the symmetry energy around saturation $\rho=\rho_{0}$ as

$$
E_{\mathrm{sym}}(\rho) \equiv E_{\mathrm{sym}}+\frac{L}{3} \frac{\rho-\rho_{0}}{\rho_{0}}+\ldots,
$$

where $E_{\text {sym }}$ is the symmetry energy at saturation (sometimes called $S$ ), and $L$ is a parameter related to the slope of $E_{\text {sym }}(\rho)$.

Using Eq. 18 we can fit the value of $E_{\text {sym }}$ and L to the calculated EoS as described in the previous section. The result is summarized in Fig. 5, where we compare the results obtained using the AV8' and AV8'+UIX Hamiltonians (red and black symbols), the various EoS giving the indicated $E_{\text {sym }}$ obtained by changing the three-neutron force model (green and blue symbols), and results obtained using the Illinois model of three-neutron force that includes three-pion rings where we have independently changed the cutoff of the intermediate- and short-range part. It is clear that within our model the correlation between L and $E_{\text {sym }}$ is very strong, and following a linear trend. Future work will need to investigate the role of the two-body force that in this work has not been changed.

From the functional form in Eq. (11), the symmetry energy and its slope at saturation $\rho_{0}$ are given by

$$
E_{\mathrm{sym}}=a+b+16
$$

and

$$
L=3(a \alpha+b \beta)
$$




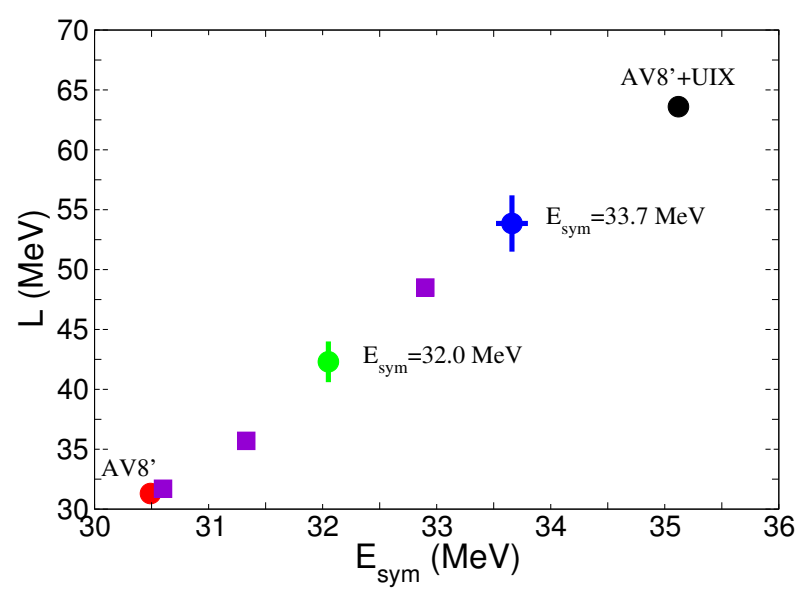

Fig. 5. The value of $\mathrm{L}$ as a function of $\mathrm{E}_{\mathrm{sym}}$ obtained from various EoS. The green and blue points with error bars correspond to the various EoS indicated by the two colored areas of Fig. 4. The red and black points show the results based on the EoS presented in Fig. 2, i.e. the results obtained using a two-body force alone and combined with the UIX model. The squared symbols correspond to results obtained by independently changing the cutoff parameters entering in $V_{R}$ and in the three-pion rings of the three-neutron force.

The parameters of Table 2 clearly show that the parameters $a$ and $\alpha$ are marginally dependent to the three-body force, and thus a precise measurement of $E_{\text {sym }}$ would in principle constrain the strength of three-nucleon interactions.

An alternative to the parametrization of Eq. (11) for the QMC results can be obtained by separating the energy of the noninteracting Fermi gas from the interaction in the following way:

$$
E\left(\rho_{n}\right)=E_{F G}+\tilde{a}\left(\frac{\rho_{n}}{\rho_{0}}\right)+\tilde{b}\left(\frac{\rho_{n}}{\rho_{0}}\right)^{2}
$$

where $E_{F G}=\frac{3}{5} \frac{\hbar^{2}}{2 m}\left(3 \pi^{2} \rho_{n}\right)^{2 / 3}$. The parameters obtained by fitting the QMC results using the above functional form are also reported in Table 2. Using this expression, the quality of the fit is slightly worse than using the expression of Eq. 11, but in this case we have only two free parameters instead of four. The symmetry energy and its slope obtained from this alternative expression can be obtained as

$$
E_{\mathrm{sym}}=E_{F G}^{0}+\tilde{a}+\tilde{b}+16
$$

and

$$
L=3\left(\frac{2}{3} E_{F G}^{0}+\tilde{a}+2 \tilde{b}\right)
$$

where $E_{F G}^{0}$ is the Fermi gas energy at density $0.16 \mathrm{fm}^{-3}$. Using the values reported in Table 2 both $E_{\text {sym }}$ and L are very close to the values reported in the same table and in Fig. 5 .

\section{Neutron star masses, radii and the EoS}

The EoS of neutron matter is the principal ingredient to study the structure of neutron stars. The neutron star matter is mainly composed by neutrons and a few protons. Once the EoS is given, the mass-radius (M-R) relation of neutron stars can be obtained by by integrating the Tolman-Oppenheimer-Volkoff (TOV) equations:

$$
\begin{gathered}
\frac{d P}{d r}=-\frac{G\left[m(r)+4 \pi r^{3} P / c^{2}\right]\left[\epsilon+P / c^{2}\right]}{r\left[r-2 G m(r) / c^{2}\right]}, \\
\frac{d m(r)}{d r}=4 \pi \epsilon r^{2},
\end{gathered}
$$

where $P=\rho^{2}(\partial E / \partial \rho)$ and $\epsilon=\rho\left(E+m_{N}\right)$ are the pressure and the energy density, $m_{N}$ is the neutron mass, $m(r)$ is the gravitational mass enclosed within a radius $r$, and $G$ is the gravitational constant. The solution of the TOV equations for a given central density gives the profiles of $\rho$, $\epsilon$ and $P$ as functions of radius $r$, and also the total radius $R$ and mass $M=m(R)$. The total radius $R$ is given by the condition $P(R)=0$. The TOV equations are modified only slightly by magnetic fields and temperatures which are expected, and rotation is less than a $10 \%$ effect for the kinds of M-R curves which we obtain below. Thus to first order, all neutron stars are expected to lie on one M-R curve determined entirely by the EoS of cold dense matter. Also, an ensemble of neutron star mass and radius measurements which determine the M-R curve constrain the EoS. The speed of sound, $c_{s}$ in the neutron star interior is $c_{s}^{2}=d P / d \epsilon$, and ensuring that this is less than the speed of light (and thus the EoS is said to be "causal") constrains the set of possible EoS. Also, the pressure must increase with increasing energy density, $d P / d \epsilon>0$, in order to ensure that the neutron star is hydrodynamically stable.

The neutron star mass measurements which provide the strongest EoS constraints are those which have the highest mass. Recent observations [31,32 have found two neutron stars almost $2 M_{\odot}$. These two data points provide some of the strongest constraints on the nature of zerotemperature QCD above the nuclear saturation density.

We begin by examining what can be deduced about the $\mathrm{M}-\mathrm{R}$ relation directly from the neutron matter EoS using the 1.97(4) $\mathrm{M}_{\odot}$ [31 measurement, but without employing a separate model for high-density matter. Using the form in Eq. (11), the energy density and pressure are given by

$$
\epsilon=\rho_{0}\left[a\left(\frac{\rho}{\rho_{0}}\right)^{1+\alpha}+b\left(\frac{\rho}{\rho_{0}}\right)^{1+\beta}+m_{n}\left(\frac{\rho}{\rho_{0}}\right)\right],
$$

and

$$
P=\rho_{0}\left[a \alpha\left(\frac{\rho}{\rho_{0}}\right)^{1+\alpha}+b \beta\left(\frac{\rho}{\rho_{0}}\right)^{1+\beta}\right] .
$$

We solve the TOV equations using QMC calculations shown in the above sections for $\rho \geq \rho_{\text {crust }}=0.08 \mathrm{fm}^{-3}$. For lower densities we use the EoS of the crust obtained in Refs. 34 and 35]. At high densities we use the maximally stiff EoS when the QMC models violate the causality and become 


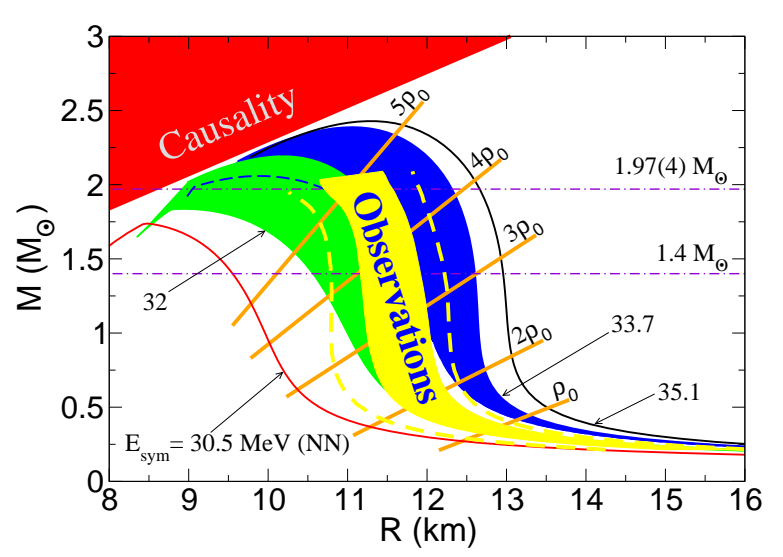

Fig. 6. The mass-radius relation for neutron stars based on the QMC neutron matter results above. Results are presented for the different EoS given in Fig. 4. The numbers indicate the value of $E_{\mathrm{sym}}$ of the various EoS. The yellow band corresponds to observations of neutron stars obtained in Ref. 33 .

superluminal. The results of the M-R diagram of neutron stars is presented in Fig. 6, where we compare the results given by the various EoS described above. The numbers in the figure indicate the symmetry energy associated with the various equations of state. In the figure we also indicate with the orange lines the density of the neutron matter inside the star. Even at large masses the radius of the neutron star is mainly governed by the equation of state of neutron matter between 1 and $2 \rho_{0}$ 36. These results are not qualitatively modified by corrections from a non-zero proton fraction 37.

The AV8' Hamiltonian alone does not support the recent observed neutron star with a mass of $1.97(4) \mathrm{M}_{\odot}$ 31. However, adding a three-body force to AV8' can provide sufficient repulsion to be consistent with all of the constraints [13]. There is a clear correlation between neutron star radii and the symmetry energy which determines the EoS of neutron matter between 1 and $2 \rho_{0}$. The results in Fig. 6also show that the most modern neutron matter EoS imply a maximum neutron star radius not larger than $13.5 \mathrm{~km}$, unless a drastic repulsion sets in just above the saturation density. This rules out EoS with large values of $L$, typical of Walecka-type mean-field models without higher-order meson couplings which can decrease $L$.

\section{Radius measurements}

Neutron star radius measurements have proven more difficult, because they require both a distance measurement and some degree of modeling of the neutron star X-ray spectrum. Low-mass X-ray binaries (LMXBs) are neutron stars accreting matter from a low mass main-sequence or white dwarf companion. There are two types of LMXB observations which have recently provided neutron star radius information. The first type are LMXBs which exhibit

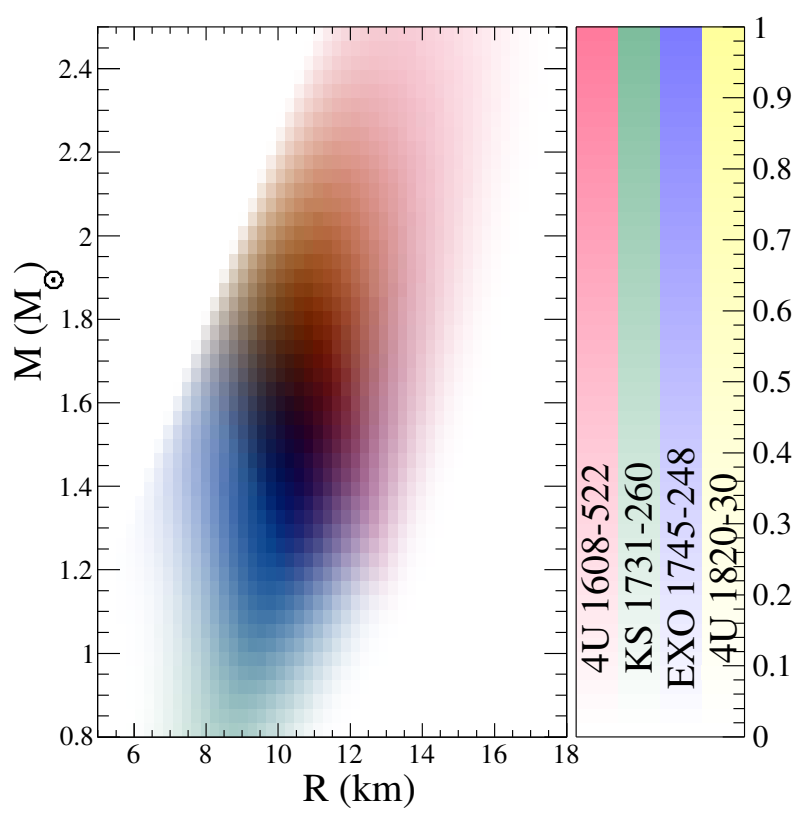

Fig. 7. Probability distributions in the mass-radius plane for four neutron stars exhibiting PRE X-ray bursts. Colors are added together in RGB color space.

photospheric radius expansion (PRE) X-ray bursts, thermonuclear explosions strong enough to temporarily lift the surface of the neutron star outwards [38, 39]. Several neutron stars have exhibited PRE X-ray bursts and four which have have been used to infer the neutron star mass and radius are given in Fig. 77, using the methods described in Ref. [0]. The second type are quiescent LMXBs, (QLMXBs), where the accretion from the companion has stopped, allowing observation of the neutron star surface which has been heated by accretion [41. A recent analysis of five neutron stars [42] including the possibility of both hydrogen and helium atmospheres and distance uncertainties is shown in Fig. 8, Note that already from these two figures alone, it is clear that the data favors a radius near $11 \mathrm{~km}$.

\section{Bayesian analysis}

In this section, we constrain the equation of state and symmetry energy using observational data sets similar to that described in Sec. 7 and include the possibility of phase transitions in matter above the nuclear saturation density. In order to do this, we parametrize the EoS of matter at higher densities with a simple expression rich enough to include exotic matter. In our fiducial model, we employ the parametrization in Eq. 11 for matter near the saturation density and treat the crust as before. At some higher density $\rho_{t} \sim 0.24-0.48 \mathrm{fm}^{-3}$ the EoS may change due to the presence of exotic matter or a higher-order manybody correction. Beginning with this density, we employ a polytrope of the form $P=K_{1} \epsilon^{\Gamma}$, fixing $K_{1}$ to ensure that the EoS is continuous and setting $\Gamma_{1}=1+1 / n_{1}$ where 


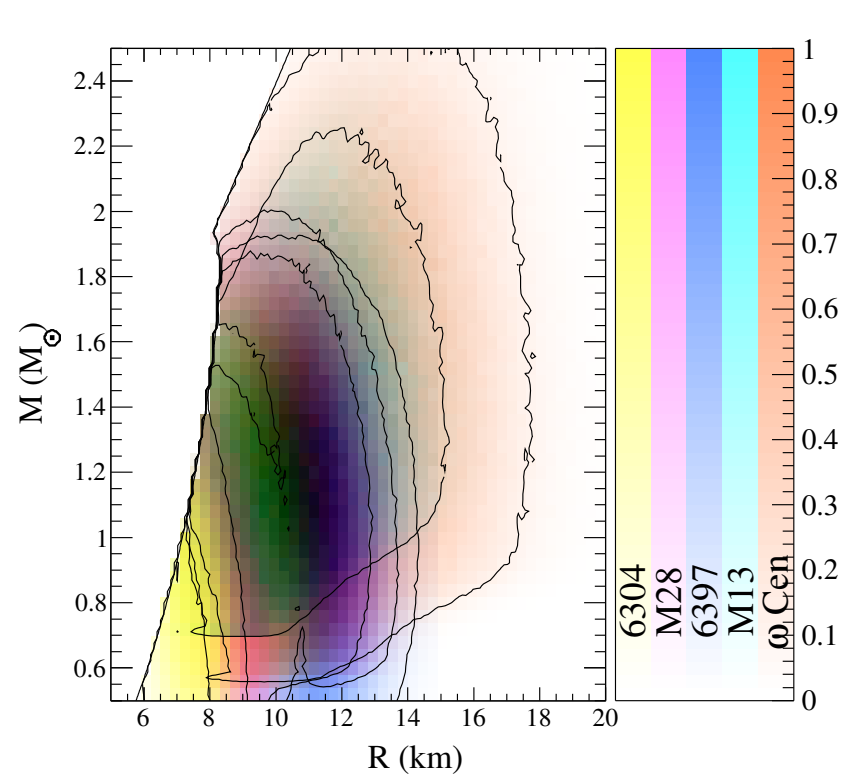

Fig. 8. Probability distributions in the mass-radius plane for five neutron stars in five globular clusters from Ref. 42. Colors are added together in RGB color space when necessary. The contour lines outline the $90 \%$ confidence regions.

$n_{1}$ is the "polytropic index". At a higher energy density, $\epsilon_{2}$, we use a second polytrope with index $n_{2}$, fixing $K_{2}$ to ensure that the EoS is continuous.

We perform a Bayesian analysis using data from QLMXB and neutron stars which exhibit PRE bursts, where our model space is given by the EoS parameters and also one parameter for the mass of each neutron star in the data set. Given an EoS, the TOV equations provide the M-R curve and thus a prediction for the radius of each neutron star from its mass. As described above, we always ensure that our EoS are causal, hydrodynamically stable, and that our M-R curves support a 1.97 solar mass star. In addition to our fiducial model, we construct a EoS parametrization which describes a hybrid neutron star with deconfined quark matter at the core. In this case, the higher-density polytrope is replaced by the quark matter model of Ref. 43 .

Finally, neutron stars contain a small amount of protons, so we multiply the EoS by a small $(\sim 10 \%)$ and density-dependent correction factor which modifies the pressure. This correction factor is obtained by averaging over Skyrme forces which give similar M-R curves to those suggested by the data.

The main results are presented in Fig. 9, where we show the M-R diagram obtained from eight neutron star data sets. In the figure, the red and black bands correspond to the M-R profile obtained within a $67 \%$ of confidence, and dashed lines correspond to $98 \%$. The red and black bands are obtained by modeling the high density part with two polytropes and with a polytrope and quark matter, respectively. Generally, the additional constraint from the observational neutron star data rules out larger

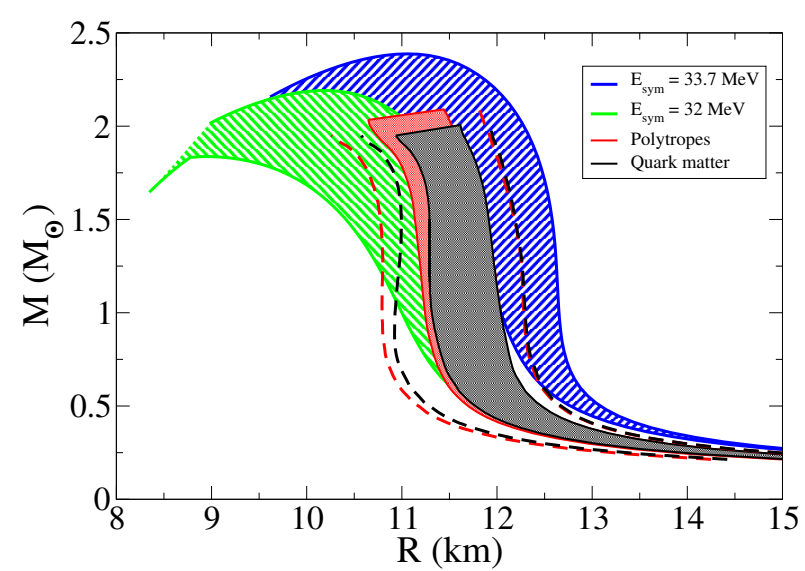

Fig. 9. The mass-radius relation obtained from the Bayesian analysis of data as described in the text. The red and black bands (dashed lines) are the results within $1 \sigma(2 \sigma)$ obtained using different models for the parametrization of the high density EoS 33 . The green and blue bands are the results presented in Fig. 6 .
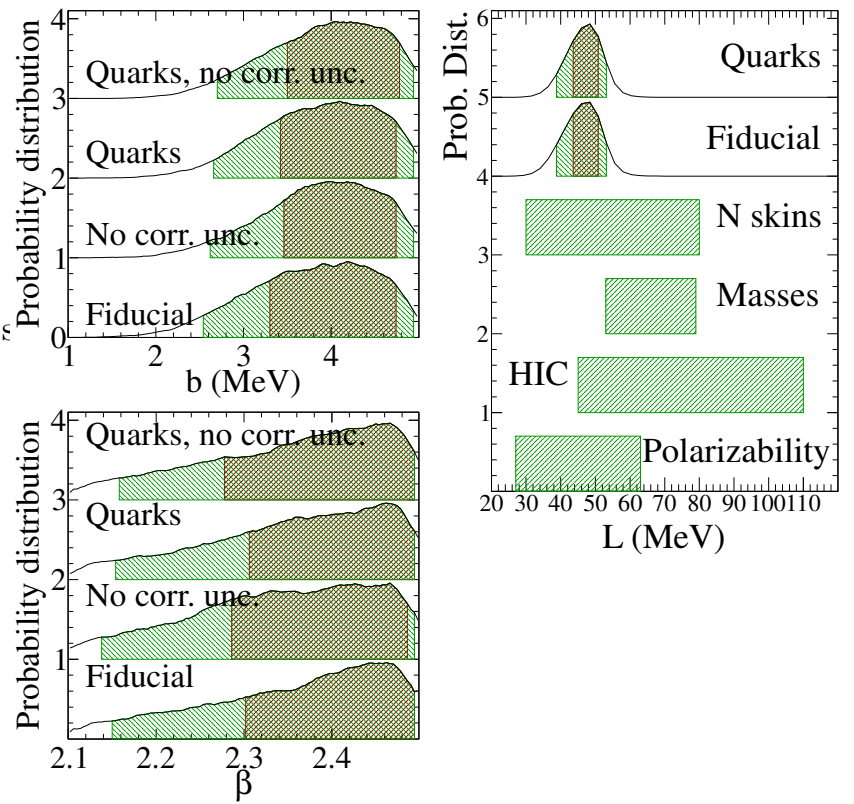

$\mathrm{L}(\mathrm{MeV})$

Fig. 10. The left panel shows probability distributions of the parameters $b$ and $\beta$ obtained from the Bayesian analysis. The right panel summarizes constraints on $L$ from observations and experiments. The top two curves show constraints on $L$ as probability distributions assuming either the fiducial model of Ref. 33. or the model containing quarks. The bottom four curves show constraints on $L$ from experiment, from neutron skins 44], nuclear masses [45], heavy-ion collisions [46, and from the electric dipole polarizability [4].

radii, bringing the maximum down to just over $12 \mathrm{~km}$ and giving a minimum down to about $10.5 \mathrm{~km}$, independent of whether or not the neutron star contains quark matter in the core. We also find that the effect of varying $\rho_{t}$ between 0.24 and $0.48 \mathrm{fm}^{-3}$ is relatively small. 


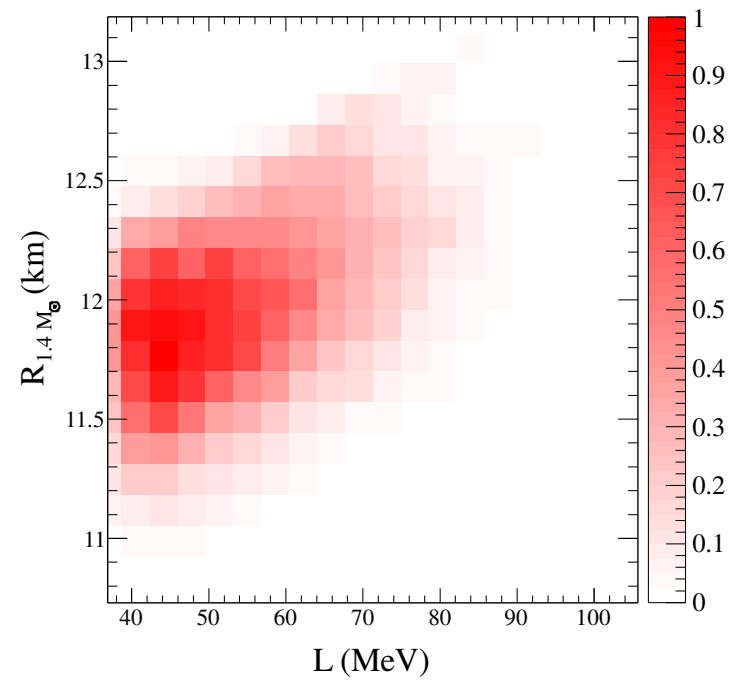

Fig. 11. Joint probability distribution of the radius of a 1.4 solar mass neutron star and $L$ obtained using the data in Figs.7 and 8

This analysis also provides posterior probability distributions for the EoS parameters. While we do not obtain significant constraints on $a$ or $\alpha$, the mass and radius data do constrain the parameters $b$ and $\beta$ (Fig. 10). While the simple parametrization employed in this section cannot fully describe the complexities of the nuclear three-body force, it does make it clear that astrophysical data is beginning to rule out some three-body forces which might otherwise be acceptable. Fig. 11 shows the joint distribution for the radius of a $1.4 \mathrm{M}_{\odot}$ neutron star and $L$. The clear positive correlation between these quantities demonstrates that the observational data on neutron star radii constrains the value of $L$. We find $32<E_{\text {sym }}<34 \mathrm{MeV}$ and $43<L<52 \mathrm{MeV}$ to within $68 \%$ confidence 33 .

\section{Conclusions}

In this paper we report on our recent efforts to constrain the symmetry energy and its density dependence by combining microscopic calculations of neutron matter and observations of neutron star structure. The EoS of pure neutron matter was calculated using Quantum Monte Carlo methods and a realistic nuclear Hamiltonian that includes the Argonne two-body force and phenomenological threebody forces. Within the Illinois/Urbana model of threeneutron forces, we have extensively explored the role of the spin-isospin-independent short-range correlations that are mostly responsible for the behavior of the EoS at high density. We have also studied the effect of the intermediateand long-range contributions of the three-body force. Within our model, we find that the uncertainty in the symmetry energy at saturation density dominates over other uncertainties associated with the short-distance structure of the three-neutron force. For the range of three nucleon forces employed we find a strong, nearly linear, correlation be- tween the symmetry energy and its slope at saturation density.

An analysis of recent astrophysical measurements of several neutron star radii is shown to provide useful constrains on the slope of the symmetry energy. To obtain agreement with neutron star radii extracted from this recent analysis we require a fairly repulsive three nucleon contribution to the energy.

We thank Steven C. Pieper for useful discussion regarding the content of this paper. The work of S.G. and J.C. is supported by the U.S. Department of Energy, Office of Nuclear Physics, by the NUCLEI SciDAC program and by the LANL LDRD program. The work of S.R. and A.W.S. is supported by DOE Grant No. DEFG02-00ER41132 and by the Topical Collaboration to study neutrinos and nucleosynthesis in hot dense matter. The work of R.B.W. is supported by the US DOE Office of Nuclear Physics under Contract No. DE-AC02-06CH11357. The computing time has been provided by Los Alamos Open Supercomputing. This research used also resources of the National Energy Research Scientific Computing Center, which is supported by the Office of Science of the U.S. Department of Energy under Contract No. DE-AC02-05CH11231.

\section{References}

1. A. W. Steiner, M. Prakash, J. M. Lattimer, and P. J. Ellis, Phys. Rep. 411, 325 (2005).

2. R. B. Wiringa, V. G. J. Stoks, and R. Schiavilla, Phys. Rev. C 51, 38 (1995).

3. S. C. Pieper and R. B. Wiringa, Annu. Rev. Nucl. Part. Sci. 51, 53 (2001).

4. E. Epelbaum, H.-W. Hammer, and U.-G. Meißner, Rev. Mod. Phys. 81, 1773 (2009).

5. N. Kaiser, European Physical Journal A 48, 135 (2012).

6. I. Tews, T. Krüger, K. Hebeler, and A. Schwenk, Phys. Rev. Lett. 110, 032504 (2013).

7. B. S. Pudliner, V. R. Pandharipande, J. Carlson, S. C. Pieper, and R. B. Wiringa, Phys. Rev. C 56, 1720 (1997).

8. S. Gandolfi, J. Carlson, and S. C. Pieper, Phys. Rev. Lett. 106, 012501 (2011).

9. J. Carlson and V. R. Pandharipande, Nucl. Phys. A 371, 301 (1981).

10. B. S. Pudliner, V. R. Pandharipande, J. Carlson, and R. B. Wiringa, Phys. Rev. Lett. 74, 4396 (1995).

11. A. Akmal, V. R. Pandharipande, and D. G. Ravenhall, Phys. Rev. C 58, 1804 (1998).

12. S. Gandolfi, A. Y. Illarionov, K. E. Schmidt, F. Pederiva, and S. Fantoni, Phys. Rev. C 79, 054005 (2009).

13. S. Gandolfi, J. Carlson, and S. Reddy, Phys. Rev. C 85, 032801 (2012).

14. Z. H. Li and H.-J. Schulze, Phys. Rev. C 78, 028801 (2008).

15. S. C. Pieper, V. R. Pandharipande, R. B. Wiringa, and J. Carlson, Phys. Rev. C 64, 014001 (2001).

16. S. C. Pieper, AIP Conf. Proc. 1011, 143 (2008).

17. S. C. Pieper, Nuclear Physics A 751, 516 (2005).

18. K. M. Nollett, S. C. Pieper, R. B. Wiringa, J. Carlson, and G. M. Hale, Phys. Rev. Lett. 99, 022502 (2007).

19. R. Schiavilla, R. B. Wiringa, S. C. Pieper, and J. Carlson, Phys. Rev. Lett. 98, 132501 (2007). 
20. A. Sarsa, S. Fantoni, K. E. Schmidt, and F. Pederiva, Phys. Rev. C 68, 024308 (2003).

21. P. Maris, J. P. Vary, S. Gandolfi, J. Carlson, and S. C. Pieper, Phys. Rev. C 87, 054318 (2013).

22. A. Gezerlis, I. Tews, E. Epelbaum, S. Gandolfi, K. Hebeler, A. Nogga, and A. Schwenk, Phys. Rev. Lett. 111, 032501 (2013)

23. K. E. Schmidt and S. Fantoni, Phys. Lett. B 446, 99 (1999).

24. J. Carlson, Phys. Rev. C 36, 2026 (1987).

25. M. M. Forbes, S. Gandolfi, and A. Gezerlis, Phys. Rev. Lett. 106, 235303 (2011).

26. J. Carlson, S. Gandolfi, K. E. Schmidt, and S. Zhang, Phys. Rev. A 84, 061602 (2011).

27. K. Hebeler and A. Schwenk, Phys. Rev. C 82, 014314 (2010).

28. M. B. Tsang, J. R. Stone, F. Camera, P. Danielewicz, S. Gandolfi, K. Hebeler, C. J. Horowitz, J. Lee, W. G. Lynch, Z. Kohley, R. Lemmon, P. Möller, T. Murakami, S. Riordan, X. Roca-Maza, F. Sammarruca, A. W. Steiner, I. Vidaña, and S. J. Yennello, Phys. Rev. C 86, 015803 (2012).

29. J. M. Lattimer and Y. Lim, ArXiv e-prints (2012).

30. P. Möller, J. R. Nix, W. D. Myers, and W. J. Swiatecki, At. Data Nucl. Data Tables 59, 185 (1995).

31. P. B. Demorest, T. Pennucci, S. M. Ransom, M. S. E. Roberts, and J. W. T. Hessels, Nature 467, 1081 (2010).

32. J. Antoniadis et al., Science 340, 448 (2013).

33. A. W. Steiner and S. Gandolfi, Phys. Rev. Lett. 108, 081102 (2012).

34. G. Baym, C. Pethick, and P. Sutherland, Astrophys. J. 170, 299 (1971).

35. J. W. Negele and D. Vautherin, Nucl. Phys. A 207, 298 (1973).

36. J. M. Lattimer and M. Prakash, Astrophys. J. 550, 426 (2001).

37. S. Gandolfi, A. Y. Illarionov, S. Fantoni, J. Miller, F. Pederiva, and K. Schmidt, Mon. Not. R. Astron. Soc. 404, L35 (2010)

38. J. van Paradijs, Astrophys. J. 234, 609 (1979).

39. F. Özel, G. Baym, and T. Güver, Phys. Rev. D 82, 101301 (2010).

40. A. W. Steiner, J. M. Lattimer, and E. F. Brown, Astrophys. J. 722, 33 (2010).

41. R. Rutledge, L. Bildsten, E. Brown, G. Pavlov, and E. Zavlin, Astrophys. J. 514, 945 (1999).

42. J. M. Lattimer and A. W. Steiner, arXiv:1305.3242 (2013).

43. M. Alford, M. Braby, M. Paris, and S. Reddy, Astrophys. J. 629, 969 (2005).

44. M. Warda, X. Viñas, X. Roca-Maza, and M. Centelles, Phys. Rev. C 80, 024316 (2009).

45. M. Liu, N. Wang, L. Z.-X., and F.-S. Zhang, Phys. Rev. C 82, 064306 (2010).

46. M. B. Tsang, Y. Zhang, P. Danielewicz, M. Famiano, Z. Li, W. G. Lynch, and A. W. Steiner, Phys. Rev. Lett. 102, 122701 (2009).

47. A. Tamii, I. Poltoratska, P. von Neumann-Cosel, Y. Fujita, T. Adachi, et al., Phys.Rev.Lett. 107, 062502 (2011). 